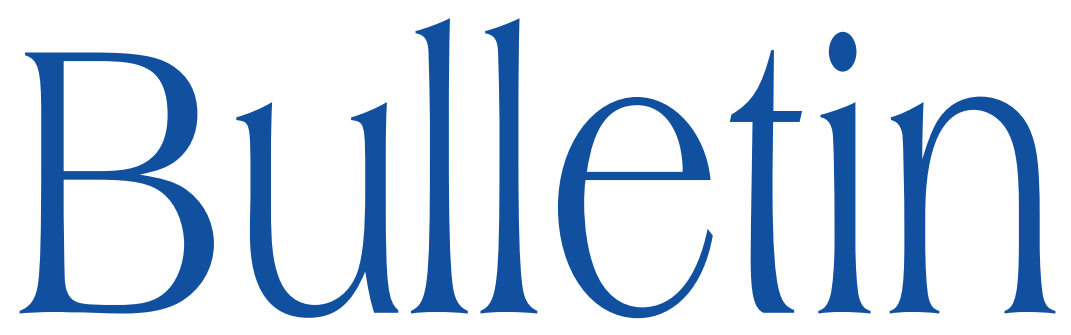

de la SOCIÉTÉ MATHÉMATIQUE DE FRANCE

\title{
SUR DES FACES DU CÔNE DE LITTLEWOOD-RICHARDSON GÉNÉRALISÉ
}

Pierre-Louis Montagard \& Nicolas Ressayre

\section{Tome 135}

Fascicule 3

2007 


\title{
SUR DES FACES DU CÔNE DE LITTLEWOOD-RICHARDSON GÉNÉRALISÉ
}

\author{
Par Pierre-Louis Montagard \& Nicolas Ressayre
}

\begin{abstract}
RÉsumé. - Soient $G \subset \hat{G}$ deux groupes réductifs connexes définis sur un corps algébriquement clos de caractéristique nulle. Notons $\mathcal{D}$ (resp. $\hat{\mathcal{D}}$ ) l'ensemble des classes d'isomorphisme des représentations irréductibles de $G$ (resp. de $\hat{G}$ ). Nous nous intéressons à l'ensemble $\mathcal{C}$ des couples $(\mu, \hat{\nu})$ dans $\mathcal{D} \times \hat{\mathcal{D}}$ pour lesquels un $\hat{G}$-module de classe $\hat{\nu}$ contient un sous- $G$-module de classe $\mu$. Il est bien connu que $\mathcal{C}$ engendre un cône polyédral dans l'espace vectoriel rationnel engendré par le produit du groupe des caractères de $G$ avec le groupe des caractères de $\hat{G}$. Par des méthodes de théorie géométrique des invariants nous étudions sous quelles conditions une inégalité linéaire définissant $\mathcal{D}$ induit une face de codimension un du cône engendré par $\mathcal{C}$. Nous appliquons ces résultats à des exemples classiques de problèmes de décompositions de représentations (produit tensoriel et pléthysme).
\end{abstract}

Texte recu le 30 octobre 2006, révisé le 31 mai 2007

Pierre-Louis Montagard, Département de Mathématiques, UMR 5149 CNRS, CC 051, Université Montpellier II, Place Eugène Bataillon, 34095 Montpellier cedex 5, France E-mail : montagar@math.univ-montp2.fr

Nicolas Ressayre, Département de Mathématiques, UMR 5149 CNRS, CC 051, Université Montpellier II, Place Eugène Bataillon, 34095 Montpellier cedex 5, France - E-mail : ressayre@math.univ-montp2.fr • Url : http://www.math.univmontp2.fr/ ${ }^{\sim}$ ressayre

Classification mathématique par sujets (2000). - 20G05.

Mots clefs. - Représentations, décomposition de représentations, cône de LittlewoodRicharson, produit tensoriel, pléthysme. 
Abstract (About some faces of the generalized Littlewood-Richardson cone)

Let $\hat{G}$ be a connected reductive algebraic group and $G$ be a reductive closed and connected subgroup of $\hat{G}$ both defined over an algebraically closed field of characteristic zero. Let $\mathcal{D}$ (resp. $\hat{\mathcal{D}}$ ) the set of isomorphism classes of irreducible representations of $G$ (resp. $\hat{G})$. We consider the set of elements $(\mu, \hat{\nu}) \in(\mathcal{D}, \hat{\mathcal{D}})$ such that an irreducible $G$-module of class $\mu$ is a submodule of a $\hat{G}$-module of class $\hat{\nu}$. This set generate a polyhedral cone $\mathcal{C}$ in the rational vector space generated by the product of characters of $G$ and $\hat{G}$. By Geometric Invariant Theory methods we give, in particular, a sufficient condition for a linear inequality defining $\mathcal{D}$ to induce a face of codimension one of $\mathcal{C}$. We apply our results to several classical example in representation theory (tensor products and plethysm).

\section{Introduction}

1.1. - Soit $k$ un corps algébriquement clos de caractéristique nulle. Soient $G \subset \hat{G}$ deux groupes algébriques réductifs connexes définis sur $k$. Soit $\hat{V}$ une représentation rationnelle de dimension finie de $\hat{G}$. Le problème général que l'on aborde ici est de décomposer $\hat{V}$ en somme de $G$-modules irréductibles. Remarquons que ce contexte recouvre de nombreux problèmes de décomposition de représentations. Citons en deux :

- si $\hat{G}=G \times G$ et si $G$ est la diagonale de $\hat{G}$, il s'agit de décomposer le produit tensoriel de deux représentations irréductibles de $G$;

- soit $G$ un groupe réductif quelconque et $\rho: G \rightarrow \operatorname{Gl}(V)$ une représentation irréductible de $G$, on peut alors poser $\hat{G}:=\mathrm{Gl}(V)$ et considérer l'inclusion $\rho(G) \subset \hat{G}$, le problème est alors de décomposer des représentations de $G$ telles que la puissance symétrique $n$-ième $S^{n} V$ de $V$, la puissance extérieure $k$-ième $\Lambda^{k} V$ de $V$, ou plus généralement de décomposer les puissances de Schur $S_{\pi} V$.

Dans ce contexte très général, on ne cherche pas à donner des formules combinatoires explicites de décomposition comme la célèbre règle de LittlewoodRichardson concernant la décomposition du produit tensoriel pour le groupe linéaire. Notre approche est plus qualitative à travers le cône de LittlewoodRichardson généralisé que nous allons définir, après avoir introduit quelques notations supplémentaires.

Nous noterons $\mathcal{D}$ (resp. $\hat{\mathcal{D}}$ ) l'ensemble des classes d'isomorphismes de représentations irréductibles de $G$ (resp. $\hat{G}$ ). Pour $\nu \in \mathcal{D}$ (resp. $\hat{\nu} \in \hat{\mathcal{D}}$ ) nous noterons $V_{\nu}\left(\right.$ resp. $\left.V_{\hat{\nu}}\right)$ une représentation irréductible de $G($ resp. $\hat{G})$ dans la classe $\nu$ (resp. $\hat{\nu})$. Si $V$ est une représentation de $G$ nous noterons $\left(V_{\nu}, V\right)$ la multiplicité de $V_{\nu}$ dans $V$, c'est-à-dire la dimension de l'espace des homomorphismes $G$-équivariants de $V_{\nu}$ vers $V$. Nous nous intéressons à l'ensemble :

$$
\mathcal{C}:=\left\{(\mu, \hat{\nu}) \in \mathcal{D} \times \hat{\mathcal{D}} \mid\left(V_{\mu}, V_{\hat{\nu}}\right) \neq 0\right\} .
$$

TOME $135-2007-\mathrm{N}^{\mathrm{O}} 3$ 
Les ensembles $\mathcal{D}$ et $\hat{\mathcal{D}}$ ont une structure naturelle de semi-groupes. De plus, M. Brion et F. Knop ont montré (voir [5]) que $\mathcal{C}$ est un sous-semigroupe de type fini de $\mathcal{D} \times \hat{\mathcal{D}}$. Dans le cas du produit tensoriel pour le groupe linéaire ce semigroupe a été appelé semi-groupe de Littlewood-Richardson (voir [17]). Comme $\mathcal{D}$ (resp. $\hat{\mathcal{D}}$ ) est en bijection avec les points entiers d'un cône d'un $\mathbb{Q}$-espace vectoriel que nous appellerons provisoirement $E$ (resp. $\hat{E}), \mathcal{C}$ engendre un cône polyédral $\tilde{\mathcal{C}}$ dans $E \times \hat{E}$. Nous appelons ce dernier cône de Littlewood-Richardson généralisé, ou plus brièvement $L R$-cône généralisé. Comme $\tilde{\mathcal{C}}$ est polyédral, il peut être défini par un nombre fini d'inégalités linéaires. La description de ces inégalités est un sujet classique spécialement dans le cas du produit tensoriel pour le groupe linéaire. Ce cas particulier qui a de multiples interprétations, voir par exemple [6], a été abordé dès 1912, avec un point de vue différent, par Hermann Weyl qui explicite dans [16] quelques inégalités satisfaites par le cône $\tilde{\mathcal{C}}$. Toujours dans ce cas particulier, la première liste complète d'inégalités a été obtenue par Klyachko dans [9].

Dans le cas général, une description complète de $\tilde{\mathcal{C}}$ a été donné par Berenstein et Sjamaar dans [2]. Récemment, Belkale et Kumar dans [1] ont considèrablement réduit la liste de ces inégalités dans le cas du produit tensoriel pour un groupe réductif quelconque.

Etant donné une liste complète d'inégalités, il est naturel de se demander si cette liste est minimale. Plus précisèment, étant donné une inéquation satisfaite par $\tilde{\mathcal{C}}$, on se demande si le cas d'égalité détermine une face de codimension un de $\tilde{\mathcal{C}}$; de telles faces seront appelées essentielles. Déterminer quelles sont les faces essentielles est une question souvent posée, voir [1], [2] ou [9], mais plus rarement abordée. On connaît cependant la liste complète des faces essentielles dans le cas du produit tensoriel de deux représentations irrréductibles du groupe linéaire (Knutson, Tao et Woodward [10]) et du groupe Spin(8) (Kapovich, Kumar et Millson [8]).

Dans le cas du produit tensoriel de $s$ représentations d'un groupe simple $G$, nos résultats montrent que les inégalités définissant les poids dominants donnent des faces essentielles de $\tilde{\mathcal{C}}$ Nous généralisons ainsi le théorème 4 de [10], obtenu pour $G=\operatorname{Sl}(n)$ et $s=2$.

1.2. - Pour décrire plus précisément nos résultats, nous introduisons maintenant quelques notations. Pour une partie $\mathcal{E}$ d'un espace vectoriel nous appellerons dimension de $\mathcal{E}$ et noterons $\operatorname{dim} \mathcal{E}$ la dimension de l'espace vectoriel engendré par $\mathcal{E}$. Soit $\mathcal{F}$ l'espace vectoriel engendré par une face du cône engendré par $\mathcal{D}$. Il induit naturellement une « face » $\mathcal{C}_{\mathcal{F}}$ de $\mathcal{C}$ définie par : $\mathcal{C}_{\mathcal{F}}:=\mathcal{C} \cap(\mathcal{F} \times \hat{E})$. Le but de cet article est d'étudier la dimension de $\mathcal{C}_{\mathcal{F}}$ et notamment de savoir si elle engendre une face essentielle de $\tilde{\mathcal{C}}$. 
Des considérations élémentaires (voir le lemme 1) permettent de montrer que la codimension de $\mathcal{C}_{\mathcal{F}}$ dans $\mathcal{C}$ est supérieure ou égale à la codimension de $\mathcal{F}$ dans $\mathcal{D}$. Cette inégalité se traduit par $\delta_{\mathcal{F}}:=\operatorname{dim} \mathcal{F}-\operatorname{dim} \mathcal{D}+\operatorname{dim} \mathcal{C}-\operatorname{dim} \mathcal{C}_{\mathcal{F}} \geq 0$. On dit que $\mathcal{F}$ est pleine si $\delta_{\mathcal{F}}=0$, c'est-à-dire si $\operatorname{dim} \mathcal{C}_{\mathcal{F}}$ est maximale. En particulier, si $\mathcal{F}$ provient d'une face essentielle de $\mathcal{D}$, alors $\delta_{\mathcal{F}}=0$ est équivalent au fait que $\mathcal{C}_{\mathcal{F}}$ soit essentielle dans $\mathcal{C}$. On peut maintenant énoncer un de nos résultats (voir le corollaire 2).

ThÉorème A. - Soit $\mathcal{F}_{1}$ et $\mathcal{F}_{2}$ deux sous-espaces de $E$ engendrés par deux faces du cône engendré par $\mathcal{D}$. Si $\mathcal{F}_{1} \subset \mathcal{F}_{2}$, alors $\delta_{\mathcal{F}_{1}} \geq \delta_{\mathcal{F}_{2}}$.

Nous présentons également dans ce travail une condition équivalente au fait que $\mathcal{F}$ soit pleine. Pour pouvoir énoncer celle-ci, nous allons introduire quelques définitions supplémentaires.

De manière classique (voir la section 3 ), on associe à $\mathcal{F}$ un groupe parabolique $P$ de $G$; le groupe $P$ se décompose en un produit semi-direct $P^{u} \rtimes L$, où $P^{u}$ est le radical unipotent de $P$ et $L$ un sous-groupe de Levi. Dans la section 4, nous montrons alors qu'il existe un sous-groupe parabolique $\hat{P}$ de $\hat{G}$ et une décomposition de Levi de celui-ci : $\hat{P}=\hat{P}^{u} \rtimes \hat{L}$ qui vérifient : $P=\hat{P} \cap G$; $P^{u}=\hat{P}^{u} \cap G$ et $L=\hat{L} \cap G$.

Nous noterons $\mathfrak{p}^{u}\left(\right.$ resp. $\left.\hat{\mathfrak{p}}^{u}\right)$, l'algèbre de Lie de $P^{u}$ (resp. $\hat{P}^{u}$ ) et $B_{L}$ (resp. $B_{\hat{L}}$ ) un sous-groupe de Borel de $L$ (resp. $\hat{L}$ ). Enfin rappelons que si un groupe algébrique $\Gamma$ agit sur une variété $X$, on appelle isotropie réductive de $\Gamma$ en $x \in X$, le quotient de $\Gamma_{x}$ par son radical unipotent. Le groupe $\hat{L}$ agit sur $\hat{\mathfrak{p}}^{u}$ par la représentation adjointe et $L \subset \hat{L}$ stabilise $\mathfrak{p}^{u} ;$ donc, $L$ agit sur $\hat{\mathfrak{p}}^{u} / \mathfrak{p}^{u}$. De plus, $\hat{L}$ et donc $L$ agissent sur $\hat{L} / B_{\hat{L}}$ par multiplication. Finalement, $L$ agit sur $\hat{\mathfrak{p}}^{u} / \mathfrak{p}^{u} \times \hat{L} / B_{\hat{L}}$ diagonalement. On peut maintenant énoncer le résultat suivant (voir le corollaire 3 ).

THÉORÈME B. - Il existe un ouvert non vide $\Omega$ de $\hat{\mathfrak{p}}^{u} / \mathfrak{p}^{u} \times \hat{L} / B_{\hat{L}}$ tel que pour tout $x \in \Omega, \delta_{\mathcal{F}}$ est égal à la différence des dimensions des isotropies réductives des groupes $L$ et $B_{L}$ en $x$.

Une conséquence immédiate des théorèmes $\mathrm{A}$ et $\mathrm{B}$ est le :

THÉORÈme C. - S'il existe un point de $\hat{G} / \hat{B}$ dont l'isotropie dans le groupe dérivé de $G$ est finie, alors toutes les faces de $\mathcal{D}$ sont pleines.

La stratégie générale, pour montrer les résultats ci-dessus est d'identifier $\mathcal{C}$ et $\mathcal{C}_{\mathcal{F}}$ à un ensemble de poids pour l'action d'un tore $T$ sur l'algèbre des fonctions régulières de $T$-variété affine $X$ et $X_{\mathcal{F}}$. Le calcul des dimensions de $\mathcal{C}$ et $\mathcal{C}_{\mathcal{F}}$ se ramène alors au calcul des dimensions des isotropies génériques de $T$ sur $X$ et $X_{\mathcal{F}}$. Nous utilisons ensuite le fait que ces dimensions sont invariantes par 
transformations birationelles et revêtement fini pour remplacer $X$ et $X_{\mathcal{F}}$ par des variétés où le calcul des isotropies génériques est plus simple.

Dans la section 6, nous appliquons nos résultats à divers exemples. Notamment nous montrons que dans des cas particuliers de pléthysme pour le groupe linéaire (i.e. $G=\mathrm{Gl}(V)$ et $\hat{G}=\mathrm{Gl}\left(S^{2} V\right)$ ou $\hat{G}=\mathrm{Gl}\left(\Lambda^{2} V\right)$ ) les conditions définissant les poids dominants de $G$ définissent des faces pleines de $\tilde{\mathcal{C}}$ et les faces essentielles de $\mathcal{D}$ induisent donc des faces essentielles de $\tilde{\mathcal{C}}$.

Remarquons enfin que $\tilde{\mathcal{C}}$ est très lié au polytope moment défini dans un cadre symplectique. La propriété d'être pleine s'interprète en terme de ces polytopes, voir la proposition 2.3.

\section{Premières propriétés}

2.1. Notations. - Commençons par quelques notations générales. Fixons $k$, un corps algébriquement clos de caractéristique nulle. Nous appelons variété, une variété algébrique quasi-projective et irréductible définie sur $k$. Si $\Gamma$ est un groupe algébrique affine sur $k$, nous noterons $\Gamma^{u}$ son radical unipotent, $[\Gamma, \Gamma]$ son groupe dérivé, $\Gamma^{\circ}$ sa composante neutre, $\Xi(\Gamma)=\operatorname{Hom}\left(\Gamma, k^{*}\right)$ le groupe de ses caractères, $\Xi_{*}(\Gamma)=\operatorname{Hom}\left(k^{*}, \Gamma\right)$ le groupe de ses sous-groupes à un paramètre et $\operatorname{Lie}(\Gamma)$ son algèbre de Lie.

Si $\Gamma$ opère algébriquement sur une variété $X$ on dit que $X$ est une $\Gamma$-variété. On note $\operatorname{Ker}(\Gamma \longrightarrow \operatorname{Aut}(X))$ le noyau de l'action de $\Gamma$ sur $X$. Si $X$ est affine et l'algèbre $k[X]^{\Gamma}$ des fonctions régulières sur $X$ invariantes par $\Gamma$ est de type fini, $X / / \Gamma$ désignera la variété affine associée à $k[X]^{\Gamma}$. L'inclusion de $k[X]^{\Gamma}$ dans $k[X]$ induit un morphisme dominant et $\Gamma$-invariant $\pi_{X}: X \longrightarrow X / / \Gamma$ que nous appelons morphisme quotient.

REMARQUe 2.1. - Notons que l'algèbre des invariants est de type fini notamment si $\Gamma$ est réductif ou si $\Gamma$ est le radical unipotent d'un sous-groupe parabolique d'un groupe réductif $G$ agissant sur $X$, (voir [7]). En particulier $k[X]^{\Gamma}$ est de type fini si $\Gamma$ est le sous-groupe unipotent maximal d'un groupe réductif $G$ agissant sur $X$.

Si $V$ est un $\mathbb{Q}$-espace vectoriel et si $\mathcal{E}$ est un sous-ensemble de $V$, nous noterons $\langle\mathcal{E}\rangle$ l'espace vectoriel engendré par $\mathcal{E}, \operatorname{dim} \mathcal{E}$ la dimension de $\langle\mathcal{E}\rangle$ et $\mathcal{E}^{\perp}$ l'ensemble des $\varphi \in V^{*}$ tels que $\varphi_{\mid \mathcal{E}}=0$. Enfin si $R$ est un groupe commutatif $R_{\mathbb{Q}}$ désignera le $\mathbb{Q}$-espace vectoriel $R \otimes_{\mathbb{Z}} \mathbb{Q}$.

Rappelons que nous considérons deux groupes algébriques réductifs connexes $G \subset \hat{G}$. Fixons, pour tout l'article, un tore maximal $T$ de $G$ et un sous-groupe de Borel $B$ de $G$. L'ensemble $\mathcal{D}$ s'identifie alors naturellement au sous-ensemble des poids dominants de $\Xi(T)$. Si $\mu \in \mathcal{D}$, nous noterons $V_{\mu}$ une représentation irréductible de poids dominant $\mu$. 
Soit $\hat{T} \subset \hat{B}$ un tore maximal et un sous-groupe de Borel de $\hat{G}$. La notation $V_{\hat{\nu}}$ désigne une représentation irréductible de $\hat{G}$ de plus haut poids $\hat{\nu}$.

2.2. Énoncé du problème. - Rappelons que nous nous intéressons à

$$
\mathcal{C}:=\left\{(\mu, \nu) \in \mathcal{D} \times \hat{\mathcal{D}} \mid\left(V_{\mu}, V_{\hat{\nu}}\right) \neq 0\right\} .
$$

Soit $\mathcal{F}$ le sous-espace vectoriel de $\Xi(T)_{\mathbb{Q}}$ engendré par une face du cône engendré par $\mathcal{D}$. Dans la suite, par abus de notation, nous appellerons $\mathcal{F}$ une face de $\mathcal{D}$. On pose alors :

$$
\mathcal{C}_{\mathcal{F}}:=\mathcal{C} \cap\left(\mathcal{F} \times \Xi_{\mathbb{Q}}(\hat{T})\right)
$$

Dans cet article, nous cherchons à comparer la codimension de $\mathcal{F}$ dans $\Xi(T)_{\mathbb{Q}}$ à celle de $\mathcal{C}_{\mathcal{F}} \operatorname{dans} \mathcal{C}$. Pour cela nous posons : $\delta_{\mathcal{F}}=\operatorname{dim} \mathcal{C}-\operatorname{dim} \mathcal{C}_{\mathcal{F}}+\operatorname{dim} \mathcal{F}-$ $\operatorname{dim} \Xi(T)$.

LEMME 1. - On a l'égalité :

$$
\operatorname{dim}\left(\langle\mathcal{C}\rangle \cap\left(\mathcal{F} \times \Xi(\hat{T})_{\mathbb{Q}}\right)\right)=\operatorname{dim}(\mathcal{C})-\operatorname{dim}(\Xi(T))+\operatorname{dim}(\mathcal{F}) .
$$

En particulier, $\delta_{\mathcal{F}} \geq 0$.

Démonstration. - Posons $d=\operatorname{dim} \mathcal{C}-\operatorname{dim}\left(\left(\mathcal{F} \times \Xi(\hat{T})_{\mathbb{Q}}\right) \cap(\langle\mathcal{C}\rangle)\right)$. Considérons l'application $\pi:\langle\mathcal{C}\rangle \rightarrow \Xi(T)_{\mathbb{Q}}$ induite par la projection de $\Xi(T) \times \hat{\Xi}(T)$ sur $\Xi(T)$ et sa transposé ${ }^{t} \pi: \operatorname{Hom}\left(\Xi(T)_{\mathbb{Q}}, \mathbb{Q}\right) \rightarrow\langle\mathcal{C}\rangle^{*}:=\operatorname{Hom}(\langle\mathcal{C}\rangle, \mathbb{Q})$.

L'entier $d$ est la dimension de l'orthogonal dans $\langle\mathcal{C}\rangle^{*}$ de $\pi^{-1}(\mathcal{F})$; ainsi, $d=$ $\operatorname{dim}\left({ }^{t} \pi\left(\mathcal{F}^{\perp}\right)\right)$. Or, comme toute représentation irréductible de $G$ apparaît dans au moins une représentation de $\hat{G}, \pi$ est surjective, donc ${ }^{t} \pi$ est injective. Ainsi, $d=\operatorname{dim}\left(\mathcal{F}^{\perp}\right)=\operatorname{dim} \Xi(T)-\operatorname{dim} \mathcal{F}$; et l'égalité du lemme est démontrée.

Comme $\operatorname{dim}\left(\langle\mathcal{C}\rangle \cap\left(\mathcal{F} \times \Xi(\hat{T})_{\mathbb{Q}}\right)\right) \geq \operatorname{dim} \mathcal{C}_{\mathcal{F}}$, on en déduit que $\delta_{\mathcal{F}} \geq 0$.

On caractérise alors le cas $\delta_{\mathcal{F}}=0$ dans la

Proposition 2.2. - On a équivalence entre :

(i) $\left\langle\mathcal{C}_{\mathcal{F}}\right\rangle=\langle\mathcal{C}\rangle \cap\left(\mathcal{F} \times \Xi(\hat{T})_{\mathbb{Q}}\right)$;

(ii) $\delta_{\mathcal{F}}=0$

On dit alors que la face $\mathcal{F}$ est pleine.

TOME $135-2007-\mathrm{N}^{\circ} 3$ 
Démonstration. - Il est clair que $\mathcal{C}_{\mathcal{F}}$ et $\left\langle\mathcal{C}_{\mathcal{F}}\right\rangle$ sont inclus dans

$$
\langle\mathcal{C}\rangle \cap\left(\mathcal{F} \times \Xi(\hat{T})_{\mathbb{Q}}\right) .
$$

Alors, l'assertion $(i)$ est équivalente à

$$
\operatorname{dim}\left\langle\mathcal{C}_{\mathcal{F}}\right\rangle=\operatorname{dim}\left(\langle\mathcal{C}\rangle \cap\left(\mathcal{F} \times \Xi(\hat{T})_{\mathbb{Q}}\right)\right) .
$$

D'après le lemme 1 ceci équivaut à $\operatorname{dim} \mathcal{C}_{\mathcal{F}}=\operatorname{dim} \mathcal{C}-\operatorname{dim} \Xi(T)+\operatorname{dim} \mathcal{F}$, soit $\delta_{\mathcal{F}}=0$.

2.3. Relations avec le polytope moment. - Soit $\hat{\nu} \in \hat{\mathcal{D}}$, posons :

$$
P_{\hat{\nu}}=\left\{\frac{\mu}{n} \in \Xi(T)_{\mathbb{Q}},(\mu, n \hat{\nu}) \in \mathcal{C}\right\} .
$$

Considérons $\hat{\mathcal{B}}:=\hat{G} / \hat{B}_{-}$la variété des drapeaux de $\hat{G}$. Alors il existe un unique fibré en droite $\hat{G}$-linéarisé $\mathcal{L}_{\hat{\nu}}$ sur $\hat{\mathcal{B}}$ tel que $\hat{B}_{-}$agisse par le caractère $-\hat{\nu}$ sur la fibre au dessus de $\hat{B}_{-} / \hat{B}_{-}$. Pour tout entier $n$, le $\hat{G}$-module des sections globales de $\mathcal{L}_{n \hat{\nu}}$ est isomorphe à $V_{n \hat{\nu}}$. Mais alors $P_{\hat{\nu}}$ est le polytope moment associé à $\mathcal{L}_{\hat{\nu}}$ pour l'action de $G$ sur $\hat{\mathcal{B}}$. Ce polytope moment est directement lié au polytope moment des géomètres symplecticiens, voir l'appendice de Mumford dans [14], et également [3]. Les polytopes moments associés aux décompositions de représentations ont été étudiés notamment dans [2], [12] et [4].

La propriété d'être pleine s'interprète aussi en termes de ces polytopes. Nous allons ici supposer que $\langle\mathcal{C}\rangle=\Xi(T)_{\mathbb{Q}} \oplus \Xi(\hat{T})_{\mathbb{Q}}$. Remarquons que cette hypothèse est vérifiée notamment lorsque $G$ est simple et non distingué dans $\hat{G}$, voir le corollaire 1 ci-après.

Proposition 2.3. - Supposons que $\langle\mathcal{C}\rangle=\Xi(T)_{\mathbb{Q}} \oplus \Xi(\hat{T})_{\mathbb{Q}} ;$ on a les résultats suivants :

(i) si $\hat{\nu}$ est un poids dominant régulier, alors $\operatorname{dim} P_{\hat{\nu}}=\operatorname{dim} \Xi(T)$;

(ii) si $\mathcal{F}$ est une face pleine de $\mathcal{D}$, alors il existe un cône convexe $\Omega \subset \hat{\mathcal{D}}$ de dimension $\operatorname{dim} \hat{\mathcal{D}}$ tel que pour tout $\hat{\nu} \in \Omega \cap \hat{\mathcal{D}}, \operatorname{dim}\left(P_{\hat{\nu}} \cap \mathcal{F}\right)=\operatorname{dim} \mathcal{F}$.

Démonstration. - Montrons (i). Posons pour tout $\hat{\nu} \in \hat{\mathcal{D}}, H_{\hat{\nu}}=\Xi(T)_{\mathbb{Q}} \times \mathbb{Q} . \hat{\nu}$ et $\tilde{P}_{\hat{\nu}}=\mathcal{C} \cap H_{\hat{\nu}}$ et soit $\tau$ la projection de $\Xi(T)_{\mathbb{Q}} \oplus \Xi(\hat{T})_{\mathbb{Q}}$ sur $\Xi(\hat{T})_{\mathbb{Q}}$. Par définition, on a $\operatorname{dim} \tilde{P}_{\hat{\nu}}=\operatorname{dim} P_{\hat{\nu}}+1$. Il suffit donc de montrer que si $\hat{\nu}$ est $\operatorname{dominant} \operatorname{dim} \tilde{P}_{\hat{\nu}}=\operatorname{dim} H_{\hat{\nu}}=\operatorname{dim} \Xi(T)+1$. Cette égalité est une conséquence du fait suivant :

si $\hat{\nu}$ est un poids dominant régulier, alors $H_{\hat{\nu}}$ rencontre l'intérieur relatif du cône convexe engendré par $\mathcal{C}$. Sinon il existe un hyperplan $H$ de $\Xi(T)_{\mathbb{Q}} \oplus \Xi(\hat{T})_{\mathbb{Q}}$ contenant $H_{\hat{\nu}}$ tel que $H$ soit un hyperplan d'appui du cône convexe engendré par $\mathcal{C}$. On vérifie alors immédiatemment que $\tau(H)$ est un hyperplan d'appui 
de $\tau(\mathcal{C})$ dans $\Xi(\hat{T})$ qui contient la droite $\mathbb{Q} . \hat{\nu}$, mais ceci contredit le fait que $\tau(\mathcal{C})=\hat{\mathcal{D}}$.

Pour (ii), remarquons d'abord que par définition : $\tilde{P}_{\hat{\nu}} \cap \mathcal{F}=\mathcal{C}_{\mathcal{F}} \cap H_{\hat{\nu}}$ et $\operatorname{dim}\left(\tilde{P}_{\hat{\nu}} \cap(\mathcal{F} \times \Xi(\hat{T}))\right)=\operatorname{dim}\left(P_{\hat{\nu}} \cap \mathcal{F}\right)+1$.

Si $\mathcal{F}$ est pleine, on a, d'après la proposition $2.2:\left\langle\mathcal{C}_{\mathcal{F}}\right\rangle=\langle\mathcal{C}\rangle \cap\left(\mathcal{F} \times \Xi(\hat{T})_{\mathbb{Q}}\right)$, et $\operatorname{donc} \operatorname{dim} \tau\left(\mathcal{C}_{\mathcal{F}}\right)=\operatorname{dim} \hat{\mathcal{D}}$. Un raisonnement élémentaire montre qu'il existe un cône convexe $\Omega$ de dimension $\operatorname{dim} \hat{\mathcal{D}}$ tel que pour tout $\hat{\nu} \in \Omega, H_{\hat{\nu}}$ rencontre l'intérieur du cone convexe engendré par $\mathcal{C}_{\mathcal{F}}$. On en déduit que pour $\hat{\nu} \in \Omega$, on $\mathrm{a}:\left\langle\tilde{P}_{\hat{\nu}} \cap(\mathcal{F} \times \Xi(\hat{T}))\right\rangle=H_{\hat{\nu}} \cap\left\langle\mathcal{C}_{\mathcal{F}}\right\rangle=H_{\hat{\nu}} \cap(\mathcal{F} \times \Xi(\hat{T}))$ et $\operatorname{donc} \operatorname{dim}\left(\tilde{P}_{\hat{\nu}} \cap(\mathcal{F} \times\right.$ $\left.\left.\Xi(\hat{T})_{\mathbb{Q}}\right)\right)=\operatorname{dim} \mathcal{F}+1$ ce qui donne bien l'égalité recherchée.

REMARQUe 2.4. - Si l'on omet l'hypothèse $\langle\mathcal{C}\rangle=\Xi(T)_{\mathbb{Q}} \oplus \Xi(\hat{T})_{\mathbb{Q}}$, le résultat de la proposition n'est plus vrai. On peut considérer l'exemple $G=H_{1} \times H_{2}$ et $\hat{G}=H_{1} \times \hat{H}_{2}$, où $H_{2}$ est un sous-groupe de $\hat{H}_{2}$ et $\mathcal{F}$ une face de la chambre dominante de $H_{1}$.

\section{Noyaux d'action d'un tore}

Considérons le sous-groupe de Levi $L$ de $G$ contenant $T$ et dont les racines sont celles de $G$ orthogonales à $\mathcal{F}$. Soit $U$ le radical unipotent de $B$ et $P$ le sous-groupe parabolique de $G$ engendré par $U$ et $L$. Posons enfin $D:=[L, L]$ et $T_{D}:=D \cap T$. Soit $\hat{U}^{-}$le radical unipotent du sous-groupe de Borel $\hat{B}^{-}$opposé à $\hat{B}$ et contenant $\hat{T}$. On montre alors la

Proposition 3.1. - (i) L'algèbre $k[\hat{G}]^{[P, P] \times \hat{U}^{-}}$est de type fini.

(ii) L'ensemble des poids de l'action de $T \times \hat{T}$ sur $k[\hat{G}]^{[P, P] \times \hat{U}^{-}}$est égal à $\mathcal{C}_{\mathcal{F}}$.

Démonstration. - Pour (i), on remarque d'abord que $[P, P]$ se décompose comme produit semi-direct $P^{u} \rtimes[L, L]$ et comme les actions de $[P, P]$ et $\hat{U}^{-}$ sur $\hat{G}$ commutent, on a égalité :

$$
k[\hat{G}]^{[P, P] \times \hat{U}^{-}}=\left(\left((k[\hat{G}])^{\hat{U}^{-}}\right)^{P^{u}}\right)^{[L, L]}
$$

Le membre de droite est une algèbre de type fini par application successive des résultats de la remarque 2.1 .

Pour (ii), d'après le théorème de Frobenius, le $\hat{G} \times \hat{G}$-module rationnel $k[\hat{G}]$ se décompose comme suit :

$$
k[\hat{G}]=\bigoplus_{\hat{\nu} \in \hat{\mathcal{D}}} V_{\hat{\nu}} \otimes V_{\hat{\nu}}^{*}
$$

TOME $135-2007-\mathrm{N}^{\mathrm{O}} 3$ 
d'où :

$$
k[\hat{G}]^{[P, P] \times \hat{U}^{-}}=\bigoplus_{\hat{\nu} \in \hat{\mathcal{D}}} V_{\hat{\nu}}^{[P, P]} \otimes V_{\hat{\nu}}^{* \hat{U}^{-}} .
$$

or $\hat{T}$ agit sur $V_{\hat{\nu}}^{* \hat{U}^{-}}$par $\hat{\nu}$. Ainsi, l'ensemble des poids de $T \times \hat{T} \operatorname{dans} k[\hat{G}]^{[P, P] \times \hat{U}^{-}}$ est égal à l'ensemble des couples $(\mu, \hat{\nu}) \in \Xi(T) \times \Xi(\hat{T})$ tels que $\hat{\nu} \in \hat{D}$ et $\mu$ est un poids de $T$ dans $V_{\hat{\nu}}^{[P, P]}$. Or par définition de $P$, l'espace $V_{\mu}^{[P, P]}$ est non nul si et seulement si $\mu$ appartient à $\mathcal{F}$ ce qui conclut la preuve.

Posons $\mathcal{C}_{\mathcal{F}}^{\vee}:=\left\{(t, \hat{t}) \in T \times \hat{T}: \forall(\mu, \nu) \in \mathcal{C}_{\mathcal{F}} \mu(t) \hat{\nu}(\hat{t})=1\right\} ;$ alors on a la

Proposition 3.2. - Le groupe $\mathcal{C}_{\mathcal{F}}^{\vee}$ est le noyau de l'action de $T \times \hat{T}$ sur la variété $[P, P] \backslash \backslash \hat{G} / / \hat{U}^{-}$.

Démonstration. - D'après la proposition $3.1, \mathcal{C}_{\mathcal{F}}^{\vee}$ est l'ensemble des couples $(t, \hat{t}) \in T \times \hat{T}$ qui agissent trivialement sur $k\left[[P, P] \backslash \backslash \hat{G} / / \hat{U}^{-}\right]$, d'où la proposition.

\section{Choix de $\hat{T}$ et $\hat{B}$}

La proposition 3.2 est vraie quelque soit le choix de $\hat{T}$ et $\hat{B}$. Dans cette section, nous allons choisir deux tels sous-groupes de $\hat{G}$ de sorte qu'il soit facile grâce à la décomposition de Bruhat de décrire un ouvert de $[P, P] \backslash \backslash \hat{G} / / \hat{U}^{-}$ stable par $T \times \hat{T}$.

Un de nos objectifs est le corollaire 2 ci-après qui compare $\delta_{\mathcal{F}_{1}}$ et $\delta_{\mathcal{F}_{2}}$ si $\mathcal{F}_{1}$ et $\mathcal{F}_{2}$ sont deux faces de $\mathcal{D}$ telles que $\mathcal{F}_{1} \subset \mathcal{F}_{2}$. Fixons les sous-groupes $T \subset B$ et les faces $\mathcal{F}_{1} \subset \mathcal{F}_{2}$. Pour $i=1,2$, nous définissons alors comme dans la section 3 les sous-groupes $T_{D_{i}}, D_{i}, L_{i}$ et $P_{i}$. L'inclusion de $\mathcal{F}_{1}$ dans $\mathcal{F}_{2}$ implique que $P_{2} \subset P_{1}$ et $L_{2} \subset L_{1}$. En revanche, $\hat{T}$ et $\hat{B}$ ne sont pas supposés donnés ici.

Soit $i=1$ ou 2. Notons $S_{i}$ le centre connexe de $L_{i}$. Considérons alors le centralisateur $\hat{L}_{i}$ de $S_{i}$ dans $\hat{G}$. Notons $\hat{D}_{i}$ le sous-groupe dérivé de $\hat{L}_{i}$ et $\hat{S}_{i}$ le centre connexe de $\hat{L}_{i}$. On a alors :

$$
\begin{array}{r}
L_{i}=\hat{L}_{i} \cap G \\
\hat{L}_{2} \subset \hat{L}_{1}
\end{array}
$$

On veut maintenant construire des sous-groupes paraboliques $\hat{P}_{i}$ de $\hat{G}$ ayant des propriétés analogues à (1). 
Pour $i=1,2$, on note $\operatorname{St}_{i}(G)$ (resp. $\left.\mathrm{St}_{i}(\hat{G})\right)$ l'ensemble des poids non triviaux de $S_{i}$ dans $\operatorname{Lie}(G)($ resp. $\operatorname{Lie}(\hat{G}))$. Pour tout $\alpha \in \Xi\left(S_{i}\right)$, on note $\mathcal{H}_{i}^{\alpha}$ le sousespace vectoriel de $\Xi_{*}\left(S_{i}\right)_{\mathbb{Q}}$ engendré par les sous-groupes à un paramètre $\lambda$ de $S_{i}$ tels que $\alpha \circ \lambda$ est trivial. Posons :

$$
C^{i}=\left\{\lambda \in \Xi_{*}\left(S_{i}\right): P_{i}=\left\{g \in G: \lim _{t \rightarrow 0} \lambda(t) g \lambda(t)^{-1} \text { existe }\right\} .\right.
$$

Alors, $C^{i}$ est l'intersection de $\Xi_{*}\left(S_{i}\right)$ et d'une composante connexe $C_{\mathbb{Q}}^{i}$ du complémentaire de $\cup_{\alpha \in \mathrm{St}_{i}(G)} \mathcal{H}_{i}^{\alpha}$ dans $\Xi_{*}\left(S_{i}\right)_{\mathbb{Q}}$. De plus, comme $P_{2} \subset P_{1}$, via l'inclusion naturelle de $\Xi_{*}\left(S_{1}\right)$ dans $\Xi_{*}\left(S_{2}\right), C_{\mathbb{Q}}^{1}$ est incluse dans l'adhérence de $C_{\mathbb{Q}}^{2}$.

Considérons $\hat{C}_{\mathbb{Q}}^{1}$ et $\hat{C}_{\mathbb{Q}}^{2}$ deux composantes connexes de $\Xi_{*}\left(S_{1}\right)_{\mathbb{Q}} \backslash \cup_{\alpha \in \operatorname{St}_{1}(\hat{G})} \mathcal{H}_{1}^{\alpha}$ et $\Xi_{*}\left(S_{2}\right)_{\mathbb{Q}} \backslash \cup_{\alpha \in \mathrm{St}_{2}(\hat{G})} \mathcal{H}_{2}^{\alpha}$ telles que $\hat{C}_{\mathbb{Q}}^{1} \subset C_{\mathbb{Q}}^{1}, \hat{C}_{\mathbb{Q}}^{2} \subset C_{\mathbb{Q}}^{2}$ et $\hat{C}_{\mathbb{Q}}^{1}$ soit incluse dans l'adhérence de $\hat{C}_{\mathbb{Q}}^{2}$. Pour $i=1,2$, on fixe $\lambda_{i}$ dans $\Xi_{*}\left(S_{i}\right) \cap \hat{C}_{\mathbb{Q}}^{i}$. Posons alors,

$$
\hat{P}_{i}=\left\{g \in \hat{G}: \lim _{t \rightarrow 0} \lambda_{i}(t) g \lambda_{i}\left(t^{-1}\right) \text { existe }\right\} .
$$

On a alors,

$$
\begin{array}{r}
\hat{P}_{2} \subset \hat{P}_{1} \\
P_{i}=\hat{P}_{i} \cap G .
\end{array}
$$

De plus, comme $\hat{P}_{i}^{u}=\left\{g \in \hat{G}: \lim _{t \rightarrow 0} \lambda_{i}(t) g \lambda_{i}\left(t^{-1}\right)=1\right\}$, on a :

$$
P_{i}^{u}=\hat{P}_{i}^{u} \cap G \text {. }
$$

Soit enfin $\hat{B}_{D_{1}}$ un sous-groupe de Borel de $\hat{D}_{1}$ contenant $B_{D_{1}}$. Posons $\hat{B}_{D_{2}}=$ $\hat{B}_{D_{1}} \cap \hat{D}_{2}$. Alors, $\hat{B}_{D_{2}}$ est un sous-groupe de Borel de $\hat{D}_{2}$. De plus, on a :

$$
\begin{array}{r}
\hat{B}_{D_{2}}=\hat{B}_{D_{1}} \cap \hat{D}_{2}, \\
B_{D_{i}}=\hat{B}_{D_{i}} \cap G .
\end{array}
$$

Soit $\hat{T}_{D_{1}}$ un tore maximal de $\hat{B}_{D_{1}}$ contenant $T_{D_{1}}$. Posons alors $\hat{T}_{D_{2}}=\hat{T}_{D_{1}} \cap$ $\hat{D}_{2}$ et $\hat{T}=\hat{S}_{1} \hat{T}_{D_{1}}=\hat{S}_{2} \hat{T}_{D_{2}}$. Alors, on a

$$
T_{D_{i}}=\left(\hat{T}_{D_{i}} \cap G\right)^{\circ} \text {. }
$$

\section{Réductions}

Nous allons dans cette section exprimer $\mathcal{C}_{\mathcal{F}_{1}}^{\vee}$ et $\mathcal{C}_{\mathcal{F}_{2}}^{\vee}$ comme noyaux d'une action du tore $T \times \hat{T}$ sur deux variétés « comparables » ce qui permettra de comparer les dimensions de $\mathcal{C}_{\mathcal{F}_{1}}$ et $\mathcal{C}_{\mathcal{F}_{2}}$. Nous rappelons d'abord plusieurs lemmes bien connus sur les actions de groupes algébriques. 


\subsection{Rappels}

LEMME 2. - Soit $G$ un groupe algébrique affine agissant sur une variété affine $X$ tels que $k[X]^{G}$ soit de type fini. Notons $\pi_{X}: X \rightarrow X / / G$ l'application quotient. Alors, se valent :

(i) $k(X)^{G}=\operatorname{Frac}\left(k[X]^{G}\right)$;

(ii) il existe un ouvert non vide $\Omega$ de $X / / G$ tel que pour $x \in \Omega, \pi_{X}^{-1}(x)$ contient une unique orbite ouverte de $G$.

On dit alors que le quotient $X / / G$ est rationnel.

Démonstration. - Voir [15, Proposition 2.5 et Proposition 3.4].

Lemme 3. - Soit $G$ un groupe algébrique affine tel que $\Xi(G)=1$ et soit $X$ une $G$-variété factorielle affine. Alors, le corps $k(X)^{G}$ des fractions rationnelles $G$-invariantes sur $X$ est égal au corps des fractions de $k[X]^{G}$.

Démonstration. - Voir [15, Theorem 3.3].

Lemme 4. - Soit $G$ un groupe algébrique tel que $\Xi(G)=1$. Soit $X$ et $Y$ deux $G$-variétés affines telles que:

(i) les algèbres $k[X]^{G}$ et $k[Y]^{G}$ sont de type fini;

(ii) le quotient $Y / / G$ est rationnel;

(iii) il existe une application $\varphi: X \rightarrow Y$, G-équivariante et birationnelle;

Alors les variétés $X / / G$ et $Y / / G$ sont birationnelles.

Démonstration. - Considèrons le diagramme commutatif suivant :

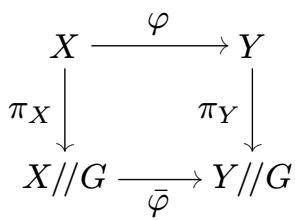

Comme $\pi_{Y}$ et $\varphi$ sont deux applications dominantes, $\bar{\varphi}$ est également dominante, donc elle induit une inclusion du corps des fractions de $k[Y]^{G}$ dans le corps des fractions de $k[X]^{G}$. Par hypothèse, $k(X)^{G}$ est égal à $k(Y)^{G}$, lui même égal au corps des fractions de $k[Y]^{G}$, le corps des fractions de $k[X]^{G}$ est donc égal au corps des fractions de $k[Y]^{G}$.

Enfin, nous terminerons par une lemme concernant l'action d'un tore sur une variété affine dont la démonstration est évidente.

Lemme 5. - Soit $T$ un tore agissant sur deux variétés $X$ et $Y$, alors on $a$ : 
(i) il existe un ouvert non vide $\Omega$ de $X$ tel que pour tout $x \in \Omega$, l'isotropie de $T$ en $x$ est égale au noyau de l'action de $T$ sur $X$;

(ii) si $f$ est un morphisme $T$-équivariant de $X$ dans $Y$ dont les fibres sont génériquement finies, alors $\operatorname{Ker}(T \longrightarrow \operatorname{Aut}(X))^{\circ}=\operatorname{Ker}(T \longrightarrow \operatorname{Aut}(Y))^{\circ}$.

5.2. - Pour $i=1,2$, on désigne par $T_{D_{i}} \subset D_{i} \subset L_{i} \subset P_{i}$ les sous-groupes associés à $\mathcal{F}_{i}$ comme dans la section 3. On a alors les inclusions suivantes : $P_{2} \subset P_{1}, L_{2} \subset L_{1}, D_{2} \subset D_{1}$ et $P_{1}^{u} \subset P_{2}^{u}$. On choisit alors $\hat{L}_{1}, \hat{L}_{2}, \hat{P}_{1}, \hat{P}_{2} \ldots$ comme dans la section 4 . Nous noterons $\mathfrak{p}_{1}^{u}:=\operatorname{Lie}\left(P_{1}^{u}\right)$ et $\hat{\mathfrak{p}}_{1}^{u}:=\operatorname{Lie}\left(\hat{P}_{1}^{u}\right)$. Remarquons que :

- le groupe $D_{1}$ agit sur $\hat{\mathfrak{p}}_{1}^{u}$ via l'action adjointe, en laissant stable $\mathfrak{p}_{1}^{u} ;$ il agit donc sur $\hat{\mathfrak{p}}_{1}^{u} / \mathfrak{p}_{1}^{u}$

- $D_{1}$ agit également par multiplication à gauche sur $\hat{L}_{1} / / \hat{U}_{D_{1}}^{-}$donc sur le produit $\hat{\mathfrak{p}}_{1}^{u} / \mathfrak{p}_{1}^{u} \times \hat{L}_{1} / / \hat{U}_{D_{1}}^{-}$.

\section{Proposition 5.1. - On a l'égalité :}

$$
\mathcal{C}_{\mathcal{F}_{2}}^{\vee}=\operatorname{Ker}\left(T \times \hat{T} \longrightarrow \operatorname{Aut}\left(\left(\hat{\mathfrak{p}}_{1}^{u} / \mathfrak{p}_{1}^{u} \times \hat{L}_{1} / / \hat{U}_{D_{1}}^{-}\right) / /\left[D_{1} \cap P_{2}, D_{1} \cap P_{2}\right]\right)\right) .
$$

En particulier (pour $\mathcal{F}_{1}=\mathcal{F}_{2}=\mathcal{F}$ ),

$$
\mathcal{C}_{\mathcal{F}}^{\vee}=\operatorname{Ker}\left(T \times \hat{T} \longrightarrow \operatorname{Aut}\left(\left(\hat{\mathfrak{p}}^{u} / \mathfrak{p}^{u} \times \hat{L} / / \hat{U}_{D}^{-}\right) / / D\right)\right) ;
$$

Enfin, pour $\mathcal{F}=\mathcal{F}_{1}$ et $\mathcal{F}_{2}=\Xi(T)_{\mathbb{Q}}$, on obtient :

$$
\mathcal{C}^{\vee}=\operatorname{Ker}\left(T \times \hat{T} \longrightarrow \operatorname{Aut}\left(\left(\hat{\mathfrak{p}}^{u} / \mathfrak{p}^{u} \times \hat{L} / / \hat{U}_{D}^{-}\right) / / U_{D}\right)\right) .
$$

Démonstration. — Rappelons que d'après la proposition 3.2

$$
\mathcal{C}_{\mathcal{F}_{2}}^{\vee}=\operatorname{Ker}\left(T \times \hat{T} \longrightarrow \operatorname{Aut}\left(\left[P_{2}, P_{2}\right] \backslash \backslash \hat{G} / / \hat{U}^{-}\right)\right)
$$

La décomposition de Bruhat de $\hat{G}$ par rapport au sous-groupe parabolique $\hat{P}_{1}$ implique que $\hat{P}_{1} \hat{P}_{1}^{u}=\hat{P}_{1}^{u} \hat{P}_{1}^{-}=\hat{P}_{1}^{u} \hat{L}_{1} \hat{P}_{1}^{u}$ est un ouvert de $\hat{G}$ stable par $P_{2} \times \hat{B}^{-}$. Le produit dans $\hat{G}$ induit les trois isomorphismes suivants : $\hat{U}^{-} \simeq$ $\hat{P}_{1}^{u-} \rtimes \hat{U}_{D_{1}}^{-},\left[P_{2}, P_{2}\right] \simeq P_{1}^{u} \rtimes\left[D_{1} \cap P_{2}, D_{1} \cap P_{2}\right]$ et $\hat{P}_{1}^{u} \hat{L}_{1} \hat{P}_{1}^{u}{ }^{-} \simeq P_{1}^{u} \times \hat{L}_{1} \times \hat{P}_{1}^{u}{ }^{-}$. En utilisant ces décompositions, on obtient les isomorphismes d'algèbres suivants :

$$
\begin{gathered}
k\left[\hat{P}_{1}^{u} \hat{L}_{1}{\hat{P_{1}^{u}}}^{-}\right]^{\left[P_{2}, P_{2}\right] \times \hat{U}^{-}} \simeq k\left[P_{1}^{u} \backslash \hat{P}_{1}^{u} \times \hat{L}_{1}\right]^{\left[D_{1} \cap P_{2}, D_{1} \cap P_{2}\right] \times \hat{U}_{D_{1}}^{-}} \\
\simeq k\left[P_{1}^{u} \backslash \hat{P}^{u} \times \hat{L}_{1} / / \hat{U}_{D_{1}}^{-}\right]^{\left[D_{1} \cap P_{2}, D_{1} \cap P_{2}\right]} .
\end{gathered}
$$

En particulier ces algèbres sont toutes de type fini. En appliquant les lemmes 4 et 5 , on en déduit :

$$
\begin{aligned}
& \mathcal{C}_{\mathcal{F}_{2}}^{\vee}=\operatorname{Ker}\left(T \times \hat{T} \longrightarrow \operatorname{Aut}\left(\left(\left(P_{1}^{u} \backslash \hat{P}_{1}^{u}\right) \times\left(\hat{L}_{1} / / \hat{U}_{D_{1}}^{-}\right)\right) / /\left[D_{1} \cap P_{2}, D_{1} \cap P_{2}\right]\right)\right) . \\
& \text { томе } 135-2007-\text { No }^{\circ}
\end{aligned}
$$


Pour finir, il suffit de remarquer que la variété $P_{1}^{u} \backslash \hat{P}_{1}^{u}$ est $D_{1}$-isomorphe à l'espace affine $\hat{\mathfrak{p}}_{1}^{u} / \mathfrak{p}_{1}^{u}$, voir [13].

Soit $\mathfrak{u}$ et $\hat{\mathfrak{u}}$ les algèbres de Lie de $U$ et $\hat{U}$. Le corollaire suivant qui se déduit d'un cas particulier de la proposition 5.1 permet de calculer la dimension de $\mathcal{C}$.

Corollaire 1. - On a les égalités :

$$
\operatorname{dim} \mathcal{C}^{\vee}=\operatorname{dim} \operatorname{Ker}(T \longrightarrow \operatorname{Aut}(\hat{\mathfrak{u}} / \mathfrak{u}))=\operatorname{dim} \operatorname{Ker}(T \longrightarrow \operatorname{Aut}(\hat{G} / G)),
$$

où $T$ agit par conjuguaison sur $\hat{\mathfrak{u}} / \mathfrak{u}$ et par multiplication à gauche sur $\hat{G} / G$. En particulier si $G$ est simple et non distingué dans $\hat{G}$, alors $\operatorname{dim} \mathcal{C}^{\vee}=0$.

Démonstration. - La première égalité se déduit directement de la proposition 5.1 appliquée à $\mathcal{F}_{1}=\mathcal{F}_{2}=\Xi(T)_{\mathbb{Q}}$.

Pour la deuxième égalité on remarque tout d'abord que :

$$
\operatorname{Ker}(T \longrightarrow \operatorname{Aut}(\hat{\mathfrak{u}} / \mathfrak{u}))=\operatorname{Ker}(T \longrightarrow \operatorname{Aut}(\hat{\mathfrak{g}} / \mathfrak{g})),
$$

où $\hat{\mathfrak{g}}, \mathfrak{g}$ sont les algèbres de Lie respectives de $\hat{G}$ et $G$ et où l'action de $T$ sur $\hat{\mathfrak{g}} / \mathfrak{g}$ est induite par l'action adjointe. D'après Luna [11], il existe un morphisme étale $T$-équivariant de $\hat{G} / G$ dans $\hat{\mathfrak{g}} / \mathfrak{g}$, et donc d'après le lemme 5 , on a égalité :

$$
\operatorname{dim} \operatorname{Ker}(T \longrightarrow \operatorname{Aut}(\hat{\mathfrak{g}} / \mathfrak{g}))=\operatorname{dim} \operatorname{Ker}(T \longrightarrow \operatorname{Aut}(\hat{G} / G)),
$$

où $T$ agit sur la variété $\hat{G} / G$ par conjuguaison. Mais cette action est égale à l'action de $T$ sur $\hat{G} / G$ par multiplication à gauche.

Pour montrer la dernière assertion, il suffit de remarquer que le noyau de $T$ sur $\hat{G} / G$ est inclus dans $\bigcap_{\hat{g} \in \hat{G}} \hat{g} G \hat{g}^{-1}$ qui est un sous-groupe distingué de $G$ et de $\hat{G}$.

5.3. - Considérons les trois applications finies :

et

$$
\begin{aligned}
\tau: S_{1} \times\left(S_{2} \cap T_{D_{1}}\right)^{\circ} \times T_{D_{2}} & \longrightarrow T \\
(s, t, u) & \longmapsto s t u \\
\hat{\tau}: \hat{S}_{1} \times \hat{T}_{D_{1}} & \longrightarrow \hat{T} \\
(s, t) & \longmapsto s t \\
\varphi: \hat{P}_{1}^{u} \times \hat{D}_{1} \times \hat{S}_{1} & \longrightarrow \hat{P}_{1}^{u} \times \hat{L}_{1} \\
(\hat{p}, \hat{d}, \hat{s}) & \longmapsto(\hat{p}, \hat{d} \hat{s})
\end{aligned}
$$

On munit $\hat{P}_{1}^{u} \times \hat{D}_{1} \times \hat{S}_{1}$ d'une action de $\hat{U}_{D_{1}}^{-} \times\left(\hat{P}_{1}^{u} D_{1}\right)$ par :

$$
(\hat{u}, p d) \cdot(\hat{p}, \hat{d}, \hat{s})=\left(p d \hat{p} d^{-1}, d \hat{d} \hat{u}^{-1}, \hat{s}\right) .
$$


On munit enfin $\hat{P}_{1}^{u} \times \hat{D}_{1} \times \hat{S}_{1}$ d'une action du tore $S_{1} \times\left(S_{2} \cap T_{D_{1}}\right)^{\circ} \times T_{D_{2}} \times$ $\hat{S}_{1} \times \hat{T}_{D_{1}}($ noté $\mathbb{T})$ par :

$$
\left(s_{1}, s_{2}, t, \hat{s}_{1}, \hat{t}_{1}\right) \cdot(\hat{p}, \hat{d}, \hat{s})=\left(s_{1} s_{2} t \hat{p} t^{-1} s_{2}^{-1} s_{1}^{-1}, s_{2} t_{1} \hat{d} \hat{t}_{1}^{-1}, s_{1} \hat{s} \hat{s}_{1}^{-1}\right) .
$$

On peut maintenant simplifier l'expression de la composante neutre de $\mathcal{C}_{\mathcal{F}_{2}}^{\vee}$ :

Proposition 5.2. - Posons $H_{2}=\left[D_{1} \cap P_{2}, D_{1} \cap P_{2}\right]$. La composante neutre de $\mathcal{C}_{\mathcal{F}_{2}}^{\vee}$ est isomorphe $\grave{a}$

$$
T_{D_{2}} \times \operatorname{Ker}\left(S_{1} \times\left(S_{2} \cap T_{D_{1}}\right)^{\circ} \times \hat{T}_{D_{1}} \longrightarrow \operatorname{Aut}\left(\left(\hat{\mathfrak{p}}_{1}^{u} / \mathfrak{p}_{1}^{u} \times \hat{D}_{1} / / \hat{U}_{D_{1}}^{-}\right) / / H_{2}\right)\right)^{\circ} .
$$

Démonstration. - Notons $\mathcal{C}_{\mathcal{F}_{2}}^{\vee, \circ}$ la composante neutre de $\mathcal{C}_{\mathcal{F}_{2}}^{\vee}$. On munit les variétés $\hat{\mathfrak{p}}_{1}^{u} / \mathfrak{p}_{1}^{u} \times \hat{L}_{1} / / \hat{U}_{D_{1}}^{-}$et $\hat{\mathfrak{p}}_{1}^{u} / \mathfrak{p}_{1}^{u} \times \hat{D}_{1} / / \hat{U}_{D_{1}}^{-} \times S_{1}$ d'une action du tore $\mathbb{T}$ grâce à $\tau$ et $\hat{\tau}$ et par passage au quotient. L'application $\varphi$ induit alors une application équivariante dont les fibres sont génériquement finies entre ces deux $\mathbb{T}$-variétés. Mais alors, la proposition 5.1 et le lemme 5 montrent que les composantes neutres de $\mathcal{C}_{\mathcal{F}_{2}}^{\vee}$ et de $\operatorname{Ker}\left(\mathbb{T} \longrightarrow \operatorname{Aut}\left(\left(\hat{\mathfrak{p}}_{1}^{u} / \mathfrak{p}_{1}^{u} \times \hat{D}_{1} / / \hat{U}_{D_{1}}^{-} \times S_{1}\right) / / H_{2}\right)\right)$ sont isomorphes.

Posons $M=\hat{\mathfrak{p}}_{1}^{u} / \mathfrak{p}_{1}^{u} \times \hat{D}_{1} / / \hat{U}_{D_{1}}^{-}$. Comme $H_{2}$ est inclus dans $D_{1},\left(\hat{\mathfrak{p}}_{1}^{u} / \mathfrak{p}_{1}^{u} \times\right.$ $\left.\hat{D}_{1} / / \hat{U}_{D_{1}}^{-} \times S_{1}\right) / / H_{2}$ est isomorphe à $M / / H_{2} \times \hat{S}_{1}$. Comme $T_{D_{2}}$ est inclus dans $H_{2}$, on en déduit que

$$
\mathcal{C}_{\mathcal{F}_{2}}^{\vee, \circ} \simeq\left(T_{D_{2}} \times \operatorname{Ker}\left(S_{1} \times\left(S_{2} \cap T_{D_{1}}\right)^{\circ} \times \hat{S}_{1} \times \hat{T}_{D_{1}} \longrightarrow \operatorname{Aut}\left(M / / H_{2} \times \hat{S}_{1}\right)\right)\right)^{\circ},
$$

puis que

$$
\mathcal{C}_{\mathcal{F}_{2}}^{\vee, \circ} \simeq T_{D_{2}} \times\left(\operatorname{Ker}\left(S_{1} \times\left(S_{2} \cap T_{D_{1}}\right)^{\circ} \times \hat{T}_{D_{1}} \longrightarrow \operatorname{Aut}\left(M / / H_{2}\right)\right)\right)^{\circ} .
$$

Pour arriver jusqu'à notre théorème principal, il nous faut maintenant décrire plus précisément le noyau apparaissant dans la proposition 5.2. Pour s'alléger de notations inutiles, dans la section 7 , nous présenterons dans un cadre plus général les résultats qui nous permettent de conclure.

THÉORÈme 5.3. - Il existe un ouvert non vide $\Omega$ de $\hat{\mathfrak{p}}_{1}^{u} / \mathfrak{p}_{1}^{u} \times \hat{D}_{1} / \hat{B}_{D_{1}}$ tel que pour tout $x$ dans $\Omega, \mathcal{C}_{\mathcal{F}_{2}}^{\vee, \circ}$ est isomorphe au produit de $T_{D_{2}}$ par la composante neutre de l'isotropie réductive en $x$ du groupe $L_{1} \cap P_{2}$.

Démonstration. - On applique la proposition 7.1 à la variété $\mathbf{M}=\hat{\mathfrak{p}}_{1}^{u} / \mathfrak{p}_{1}^{u} \times$ $\hat{D}_{1} / / U_{\hat{D}_{1}}^{-}$, au tore $\mathbf{T}=S_{1} \times T_{D_{1}}$, au groupe semi-simple $\mathbf{D}=D_{1}$ et au sousgroupe parabolique $\mathbf{P}=P_{2} \cap D_{1}$. Remarquons qu'alors $L_{2} \cap D_{1}$ est un Levi de $\mathbf{P}$ et que son centre connexe est égal à $\mathbf{S}=S_{2}$. On obtient l'existence d'un ouvert non vide $\Omega$ de $\hat{\mathfrak{p}}_{1}^{u} / \mathfrak{p}_{1}^{u} \times \hat{D}_{1} / / U_{\hat{D}_{1}}^{-}$tel que pour tout $x \in \Omega$ :

$\operatorname{Ker}\left(S_{1} \times\left(S_{2} \cap T_{D_{1}}\right)^{\circ} \times \hat{T}_{D_{1}} \longrightarrow \operatorname{Aut}\left(M / / H_{2}\right)\right)=\rho\left(\left(\left(P_{2} \cap D_{1}\right) \times S_{1} \times T_{D_{1}}\right)_{x}\right)$. 
Remarquons que puisque $\hat{D}_{1} / / U_{\hat{D}_{1}}^{-}$contient $\hat{D}_{1} / U_{\hat{D}_{1}}^{-}$comme ouvert, quitte à rétrécir $\Omega$, on peut supposer que l'égalité ci-dessus est vrai pour tout $x \in \Omega \subset$ $\hat{\mathfrak{p}}_{1}^{u} / \mathfrak{p}_{1}^{u} \times \hat{D}_{1} / U_{\hat{D}_{1}}^{-}$. On applique ensuite la proposition 7.2 , avec $\mathbf{D} \subset \hat{\mathbf{D}}=\hat{D}_{1}$ et $\mathbf{M}=\hat{\mathfrak{p}}_{1}^{u} / \mathfrak{p}_{1}^{u} \times \hat{D}_{1} / U_{\hat{D}_{1}}$. On obtient alors que pour $x=(a, b) \in \hat{\mathfrak{p}}_{1}^{u} / \mathfrak{p}_{1}^{u} \times \hat{D}_{1} / U_{\hat{D}_{1}}$, $\rho\left(\left(\left(P_{2} \cap D_{1}\right) \times S_{1} \times T_{D_{1}}\right)_{(a, b)}\right)$ est isomorphe au quotient de $\left(P_{2} \cap D_{1} \times S_{1}\right)_{(a, q(b))}$ par son radical unipotent, où $q$ est l'application quotient de $\hat{D}_{1} / U_{\hat{D}_{1}}^{-}$dans $\hat{D}_{1} / B_{\hat{D}_{1}}^{-}$. Le théorème suit, en remarquant que $\left(P_{2} \cap D_{1}\right) \times S_{1} \simeq P_{2} \cap L_{1}$.

Corollaire 2. - Avec les notations ci-dessus,

$$
\delta_{\mathcal{F}_{1}} \geq \delta_{\mathcal{F}_{2}}
$$

Démonstration. - Comme $\operatorname{dim} \mathcal{F}_{1}-\operatorname{dim} \mathcal{F}_{2}=\operatorname{dim} T_{D_{2}}-\operatorname{dim} T_{D_{1}}$, on a $\delta_{\mathcal{F}_{1}}-$ $\delta_{\mathcal{F}_{2}}=\left(\operatorname{dim} \mathcal{C}_{\mathcal{F}_{1}}^{\vee}-\operatorname{dim} T_{D_{1}}\right)-\left(\operatorname{dim} \mathcal{C}_{\mathcal{F}_{2}}^{\vee}-\operatorname{dim} T_{D_{2}}\right)$. Mais alors, le corollaire découle immédiatement du théorème 5.3 appliqué une fois à la paire de face $\left(\mathcal{F}_{1}, \mathcal{F}_{2}\right)$ et une fois à la paire $\left(\mathcal{F}_{1}, \mathcal{F}_{1}\right)$.

Corollaire 3. - Il existe un ouvert non vide $\Omega$ de $\hat{\mathfrak{p}}^{u} / \mathfrak{p}^{u} \times \hat{D} / \hat{B}_{D}$ tel que pour tout $x$ dans $\Omega, \delta_{\mathcal{F}}$ est égal à la différence des dimensions des isotropies réductives des groupes $L$ et $B_{L}$ en $x$.

Démonstration. - Il suffit d'appliquer le théorème 5.3 une première fois à la paire de faces $(\mathcal{F}, \mathcal{F})$ et ensuite à la paire $\left(\mathcal{F}, \Xi(T)_{\mathbb{Q}}\right)$.

Sur les exemples, le théorème 5.3 sera souvent utilisé via le corollaire suivant :

Corollaire 4. - S'il existe un point de $\hat{\mathfrak{p}}^{u} / \mathfrak{p}^{u} \times \hat{D} / \hat{B}_{D}$ dont l'isotropie dans $D$ est finie, alors $\mathcal{F}$ est pleine. En particulier, s'il existe un point de $\hat{G} / \hat{B}$ dont l'isotropie dans le groupe dérivé de $G$ est finie, alors toutes les faces de $\mathcal{D}$ sont pleines.

Démonstration. - Soit $\Omega$ un ouvert de $\hat{\mathfrak{p}}^{u} / \mathfrak{p}^{u} \times \hat{D} / \hat{B}_{D}$ vérifiant le corollaire 3 . Comme l'ensemble des points dont l'isotropie pour $D$ est fini est un ouvert, il existe $x \in \Omega$ tel que $D_{x}$ soit fini. Mais alors, $L_{x}^{\circ}$ est inclus dans le centre de $L$ et donc dans $B_{L}$ et $\mathcal{F}$ est pleine. S'il existe $x \in \hat{G} / \hat{B}$ dont l'isotropie pour $D$ est finie, nous venons de montrer que la face $\{0\}$ est pleine. Mais alors, le corollaire 2 montre que toutes les faces sont pleines. 


\section{Exemples}

Exemple 1. Le produit tensoriel.

Proposition 6.1. - Soit $G$ un groupe simple inclus diagonalement dans $\hat{G}=$ $G^{s}$ le produit de $s$ copies de $G$.

(i) Pour $s=2$ toutes les faces non réduites à un point sont pleines. De plus, $\delta_{\{0\}}$ est égal au rang de $G$.

(ii) Si $s>2$ alors toutes les faces sont pleines.

Démonstration. - Fixons un sous-groupe de Borel $B$ de $G$ et un tore maximal $T$ de $B$. Supposons $s=2:$ soit $\mathcal{F}$ une face de l'ensemble des poids dominants de $(G, B, T)$. Notons $P$ le sous-groupe parabolique de $G$ associé à la face $\mathcal{F}$, $L$ son sous-groupe de Levi contenant $T$ et enfin $D$ le sous-groupe dérivé de $L$. Posons :

$$
B_{D}=B \cap D, \quad \hat{P}=P \times P, \hat{D}=D \times D, \quad \hat{B}=B \times B, \quad \hat{B}_{D}=B_{D} \times B_{D} .
$$

Alors, $\hat{B}_{D} \subset \hat{D} \subset \hat{P} \subset \hat{G}$ satisfont aux propriétés de la section 4. De plus, $M=\mathfrak{p}^{u} \times D / B_{D} \times D / B_{D}$. En particulier, l'isotropie générique $I$ de $D$ agissant sur $M$ est égale à celle de $T_{D}$ agissant sur $\mathfrak{p}_{u}$. Soit $P^{-}$le sous-groupe parabolique contenant $T$ et opposé à $P$. Comme $\mathfrak{p}_{u}$ est isomorphe à un ouvert $T$-stable de $G / P^{-}, I$ est égale au noyau de l'action de $T_{D}$ dans $G / P^{-}$, c'est-à-dire à $\bigcap_{g \in G} g P^{-} g^{-1} \cap T_{D}$. Comme $G$ est simple cette dernière est finie à moins que $P^{-}=G$, c'est-à-dire à moins que $\mathcal{F}=\{0\}$.

Supposons maintenant que $\mathcal{F}=\{0\}$. Alors, $M=G / B \times G / B$. Donc, l'isotropie générique de $D=G$ sur $M$ est un tore maximal de $G$ alors que celle de $B$ est finie. Le corollaire 3 achève alors la démonstration.

Si $s>2$, d'après le corollaire 4 et puisque $G$ est simple, il suffit de montrer qu'il existe un point de $\hat{G} / \hat{B}=(G / B)^{s}$ tels que l'isotropie dans $G$ soit finie. Mais génériquement cette isotropie est incluse dans l'isotropie du tore $T$ dans $G / B$, et celle-ci est finie en utilisant le même type d'argument que précédemment.

REMARQUe 6.2. - Le calcul de $\delta_{\{0\}}$ pour $s=2$ peut se faire directement en utilisant la définition de $\mathcal{C}_{0}$.

Exemple 2. Soit $V$ un $k$-espace vectoriel de dimension au moins deux. Considérons le morphisme $i: \mathrm{Sl}(V) \rightarrow \mathrm{Sl}\left(S^{2} V\right)$. On a :

Proposition 6.3. - Pour l'inclusion de $G=i(\operatorname{Sl}(V))$ dans $\hat{G}=\operatorname{Sl}\left(S^{2} V\right)$, toutes les faces sont pleines.

D'après le corollaire 4 , il suffit de montrer le

TOME $135-2007-\mathrm{N}^{\mathrm{O}} 3$ 
Lemme 6. - Il existe un drapeau complet de $S^{2} V$ dont l'isotropie pour $\mathrm{Sl}(V)$ est finie.

Démonstration. - Nous allons construire un drapeau complet $\Omega$ de $S^{2} V^{*}$ dont le groupe d'isotropie $H$ est fini. Son orthogonal vérifiera la propriété du lemme. Soit $\mathcal{B}=\left(e_{1}, \ldots, e_{n}\right)$ une base de $V$ et $\left(e_{1}^{*}, \ldots, e_{n}^{*}\right)$ sa base duale, le premier sous-espace du drapeau $\Omega$ est la droite engendrée par $\omega_{0}=\Sigma e_{i}^{* 2} \in S^{2} V^{*}$. La composante neutre du groupe d'isotropie de cette droite est $\mathrm{SO}(V)$. Il suffit donc de montrer que l'intersection $H \cap \mathrm{SO}(V)$ est finie. Si $\omega$ est un élément quelconque de $S^{2} V^{*}$, on définit :

$$
\begin{aligned}
\tilde{\omega}: V & \rightarrow V^{*} \\
x & \mapsto \omega(x, .) .
\end{aligned}
$$

Comme $\omega_{0}$ est non dégénérée, $\tilde{\omega}_{0}$ est un isomorphisme $\mathrm{SO}(V)$-équivariant. Ce qui nous permet de définir une application :

$$
\begin{aligned}
\varphi: S^{2} V^{*} & \rightarrow \operatorname{End}(V) \\
\omega & \mapsto \tilde{\omega}_{0}^{-1} \circ \tilde{\omega} .
\end{aligned}
$$

L'application $\varphi$ est un isomorphisme linéaire $\mathrm{SO}(V)$-équivariant (l'action sur l'espace de droite est donnée par la conjugaison). Soit $\omega_{1}=\Sigma i e_{i}^{* 2}$. On voit alors que $\varphi\left(\omega_{0}\right)$ est l'identité de $V$ et que $e_{i}$ est vecteur propre de $\varphi\left(\omega_{1}\right)$ pour la valeur propre $i$. Le stabilisateur du drapeau $\left\langle\omega_{0}\right\rangle \subset\left\langle\omega_{0}, \omega_{1}\right\rangle$ dans $\mathrm{SO}(V)$ est alors composé des éléments $g \in \mathrm{SO}(V)$ tel qu'il existe $a, b \in k$ vérifiant $g . \varphi\left(\omega_{1}\right)=\varphi\left(a \omega_{0}+b \omega_{1}\right)$. La matrice de $\varphi\left(a \omega_{0}+b \omega_{1}\right)$ dans $\mathcal{B}$ est une matrice diagonale de valeurs propres $\{a+b i: i=1, \ldots, n\}$. Cet ensemble est égal à l'ensemble des valeurs propres de $g . \varphi\left(\omega_{1}\right)$ (donc de $\varphi\left(\omega_{1}\right)$ soit $\{1, \ldots, n\}$ ). On en déduit que ou bien $a=0$ et $b=1$, ou bien $a=1+n$ et $b=-1$; puis que la composante neutre du groupe d'isotropie de $\left\langle\omega_{0}\right\rangle \subset\left\langle\omega_{0}, \omega_{1}\right\rangle$ est inclus dans l'ensemble des matrices diagonales. Comme par ailleurs elle est inclus dans $\mathrm{SO}(V)$, elle est triviale. Le lemme suit alors facilement.

Exemple 3. Nous allons ici regarder l'exemple donné par le morphisme $i$ : $\mathrm{Sl}(V) \rightarrow \mathrm{Sl}\left(\Lambda^{2} V\right)$. On pose alors $G=i\left(\mathrm{Sl}(V)\right.$ et $\hat{G}=\mathrm{Sl}\left(\Lambda^{2} V\right)$. Si $\operatorname{dim} V=2$, alors $\Lambda^{2} V \simeq k$ et $\hat{G}$ est trivial; si $\operatorname{dim} V=3$, alors $\Lambda^{2} V \simeq V^{*}$ et $G=\hat{G}$. On suppose donc que $\operatorname{dim} V \geq 4$.

Proposition 6.4. - Si $\operatorname{dim} V \geq 4$, alors pour l'inclusion de $G=i(\operatorname{Sl}(V))$ dans $\hat{G}=\operatorname{Sl}\left(\Lambda^{2} V\right)$, toutes les faces de $\mathcal{D}$ sont pleines.

Comme dans l'exemple précédent, la proposition est une conséquence du Lemme 7. - Si $\operatorname{dim} V \geq 4$, alors il existe un drapeau de $\Lambda^{2}\left(V^{*}\right)$ dont l'isotropie dans $\mathrm{Sl}(V)$ est finie. 
Démonstration. - Dans $\Lambda^{2}\left(V^{*}\right)$, il existe un élément non dégénéré si et seulement si $\operatorname{dim} V$ est paire. Commençons par le cas où $\operatorname{dim} V$ est paire. On pose $\operatorname{dim} V=2 p$ et on fixe une base $\mathcal{B}=\left(e_{1}, \ldots, e_{p}, \varepsilon_{1}, \ldots, \varepsilon_{p}\right)$ de $V$ et $\mathcal{B}^{*}=$ $\left(e_{1}^{*}, \ldots, e_{p}^{*}, \varepsilon_{1}^{*}, \ldots, \varepsilon_{p}^{*}\right)$ sa base duale. On pose $\omega_{0}=\Sigma_{i=1}^{p} e_{i}^{*} \wedge \varepsilon_{i}^{*}$. Soit $H_{0}$ la composante neutre de l'isotropie de la droite engendrée par $\omega_{0}$. Comme $H_{0} \subset \mathrm{Sl}(V)$, on voit que $H_{0}=\operatorname{Sp}\left(\omega_{0}\right)$. Comme dans la démonstration du lemme 6 , on définit pour tout $\omega \in \Lambda^{2} V^{*}$ une application $\tilde{\omega}: V \longrightarrow V^{*}, x \longmapsto \omega(x,$.$) .$ On définit l'application linéaire, $\operatorname{Sp}\left(\omega_{0}\right)$-équivariante $\varphi: \Lambda^{2} V^{*} \longrightarrow \operatorname{End}(V)$, $\omega \longmapsto \tilde{\omega}_{0}^{-1} \circ \tilde{\omega}$.

Soit $\omega_{1}=\Sigma_{i=1}^{p} i e_{i}^{*} \wedge \varepsilon_{i}^{*} ; \varphi\left(\omega_{1}\right)$ admet $i=1, \ldots, p$ comme valeurs propres, et $\left(e_{i}^{*}, \varepsilon_{i}^{*}\right)$ engendre le sous-espace propre associé à $i$. Par un raisonnement analogue à l'exemple précédent, on montre alors que la composante neutre du sous-groupe d'isotropie du drapeau : $\left\langle\omega_{0}\right\rangle \subset\left\langle\omega_{0}, \omega_{1}\right\rangle$ est l'ensemble des matrices de $\operatorname{Sp}(V)$ préservant les sous-espaces $W_{i}:=\left\langle e_{i}^{*}, \varepsilon_{i}^{*}\right\rangle$. Cette composante neutre est donc isomorphe au produit : $\prod_{i=1}^{p} \mathrm{Sl}\left(W_{i}\right)$. Sous l'action de ce groupe, l'espace $\Lambda^{2} V^{*}$ se décompose en représentations irréductibles :

$$
\Lambda^{2} V^{*}=\bigoplus_{i=1}^{p} \Lambda^{2} W_{i} \oplus \bigoplus_{1 \leq i<j \leq p} W_{i} \otimes W_{j} .
$$

La suite de la construction du drapeau se fait en considérant dans l'espace dual $\Lambda^{2}\left(V^{*}\right)^{*} \simeq \Lambda^{2} V$ des vecteurs $\omega_{3}^{*}, \omega_{4}^{*}, \ldots$ dont l'orthogonal dans $\Lambda^{2} V^{*}$ contient $\left\langle\omega_{0}, \omega_{1}\right\rangle$. À cause de la décomposition ci-dessus, de tels vecteurs peuvent être pris dans $\bigoplus_{1 \leq i<j \leq p} W_{i}^{*} \otimes W_{j}^{*}$. Grâce à cette décomposition, on peut également supposer que $p=2$. Pour $\operatorname{Sl}(W) \times \mathrm{Sl}(W)$, l'espace $W \otimes W$ est isomorphe à l'espace $\operatorname{End}(W)$. À indice fini près l'isotropie de la droite engendrée par l'application identité est le groupe $\mathrm{Sl}(W)$ inclus diagonalement dans $\operatorname{Sl}(W) \times \operatorname{Sl}(W)$ et qui agit sur $\operatorname{End}(W)$ par conjugaison. Comme $\operatorname{Sl}(W)$ représentation $\operatorname{End}(W)$ se décompose comme la somme de la représentation triviale et de la représentation adjointe. Le problème se ramène donc à exhiber un drapeau de cette représentation adjointe d'isotropie finie. Soit $H$ et $F$ les éléments suivants :

$$
H=\left(\begin{array}{cc}
1 & 0 \\
0 & -1
\end{array}\right), \quad F=\left(\begin{array}{ll}
0 & 1 \\
1 & 0
\end{array}\right)
$$

(dans une base quelconque de $\mathrm{W}$ ). Alors il est immédiat que le drapeau suivant : $\langle H\rangle \subset\langle H, F\rangle$ a une isotropie finie dans $\operatorname{Sl}(W)$.

Supposons maintenant que la dimension de $V$ est impaire et égale à $2 p+1$, avec $p \geq 2$. Soit $\mathcal{B}=\left(e_{1}, \ldots, e_{p}, \varepsilon_{1}, \ldots, \varepsilon_{p}, \kappa\right)$ une base de $V$. Nous noterons $W$ le sous-espace vectoriel de $V$ de base $\left(e_{1}, \ldots, e_{p}, \varepsilon_{1}, \ldots, \varepsilon_{p}\right)$.

Rappelons qu'on a une injection $\operatorname{Gl}(V)$-équivariante de $\Lambda^{2} V^{*}$ dans $\operatorname{Hom}\left(V^{*}, V\right)$. Soient $\omega_{0}, \omega_{1}$ et $\omega_{2}$ les éléments $\operatorname{de} \operatorname{Hom}\left(V^{*}, V\right)$ dont les 
matrices dans les bases $\mathcal{B}^{*}, \mathcal{B}$ sont :

$$
\omega_{0}=\left(\begin{array}{ccc}
0 & I & 0 \\
-I & 0 & 0 \\
0 & 0 & 0
\end{array}\right), \quad \omega_{1}=\left(\begin{array}{ccc}
0 & D^{t} V \\
-D & 0 & 0 \\
-V & 0 & 0
\end{array}\right), \quad \omega_{2}=\left(\begin{array}{ccc}
0 & D^{\prime} & 0 \\
-D^{\prime} & 0 & { }^{t} V \\
0 & -V & 0
\end{array}\right)
$$

où $I$ désigne la matrice identité de taille $p \times p, D$ la matrice diagonale avec comme termes diagonaux : $1,2, \ldots, p, V$ le vecteur $(1, \ldots, 1) \in k^{p}$ et $D^{\prime}$ une matrice de $\mathrm{M}_{p}(k)$ telle que les matrices $I, D, D^{\prime}$ soient linéairement indépendantes. Nous allons maintenant définir un hyperplan de $\Lambda^{2} V^{*}$ contenant les vecteurs $\omega_{0}, \omega_{1}$ et $\omega_{2}$ ce qui revient à définir une droite de $\Lambda^{2} V^{* *}$, qui s'identifie à $\Lambda^{2} V$ et donc à un sous-espace de $\operatorname{Hom}\left(V, V^{*}\right)$. Remarquons que la dualité entre $\Lambda^{2} V$ et $\Lambda^{2} V^{*}$ est la restriction de l'application bilinéaire suivante :

$$
\begin{aligned}
\operatorname{Hom}\left(V, V^{*}\right) \times \operatorname{Hom}\left(V^{*}, V\right) & \longrightarrow \quad k \\
(A, B) & \longmapsto \operatorname{trace}(B \circ A)
\end{aligned}
$$

Soit $\theta \in \operatorname{Hom}\left(V^{*}, V\right)$ défini par :

$$
\left(\begin{array}{ccc}
0 & U & 0 \\
-U & 0 & 0 \\
0 & 0 & 0
\end{array}\right)
$$

avec $U$ une matrice telle que $\operatorname{tr}(U)=\operatorname{tr}(U D)=\operatorname{tr}\left(U D^{\prime}\right)=0$, (ce choix est possible puisque $p \geq 2$ et donc $\left.\operatorname{dim} \mathrm{M}_{p}(k) \geq 4\right)$. On a alors que $\left\langle\omega_{0}, \omega_{1}, \omega_{2}\right\rangle \subset$ $\operatorname{ker} \theta$. Considérons donc le drapeau partiel :

$$
\Omega=\left(\left\langle\omega_{0}\right\rangle \subset\left\langle\omega_{0}, \omega_{1}\right\rangle \subset\left\langle\omega_{0}, \omega_{1}, \omega_{2}\right\rangle \subset \operatorname{ker} \theta\right) .
$$

Comme $\operatorname{ker} \theta=k \kappa^{*}$ et $\operatorname{ker} \omega_{0}=k . \kappa$ le groupe d'isotropie de ce drapeau est inclus dans le groupe $\mathrm{Gl}(W) \times \mathrm{Gl}(k . \kappa)$. Ce groupe respecte la décomposition $\Lambda^{2} V^{*}=\Lambda^{2} W^{*} \oplus W^{*} \otimes k . \kappa^{*}$. Les éléments $\omega_{0}$ et $\omega_{1}$ s'écrivent dans $\Lambda^{2} V^{*}$ :

$$
\omega_{0}=\Sigma_{i=1}^{p} e_{i}^{*} \wedge \varepsilon_{i}^{*} \text { et } \omega_{1}=\Sigma_{i=1}^{p} i e_{i}^{*} \wedge \varepsilon_{i}^{*}+\kappa^{*} \wedge\left(e_{1}^{*}+\cdots+e_{n}^{*}\right)
$$

On en déduit qu'un élément du stabilisateur dans $\mathrm{Gl}(W) \times \mathrm{Gl}(k \kappa)$ du drapeau : $\left\langle\omega_{0}\right\rangle \subset\left\langle\omega_{0}, \omega_{1}\right\rangle$ stabilise le drapeau de $\Lambda^{2} W^{*}:\left\langle\omega_{0}\right\rangle \subset\left\langle\omega_{0}, \Sigma i e_{i}^{*} \wedge \varepsilon_{i}^{*}\right\rangle$ et la droite engendrée par $\kappa^{*} \wedge\left(e_{1}^{*}+\cdots+e_{n}^{*}\right)$. En utilisant cette propriété et le cas de la dimension paire, on en déduit que la composante neutre du groupe d'isotropie du drapeau $\mathcal{D}$ est inclus dans le groupe composé des matrices de la forme :

$$
\left(\begin{array}{ccc}
\lambda^{p+1} I & 0 & 0 \\
Z & \lambda^{-p} I & 0 \\
0 & 0 & \lambda^{-p}
\end{array}\right)
$$

où $\lambda \in k^{*}$ et $Z$ est une matrice de taille $p \times p$ diagonale. Ensuite on écrit matriciellement que de tels éléments envoient $\omega_{2}$ dans $\left\langle\omega_{0}, \omega_{1}, \omega_{2}\right\rangle$. En utilisant 
notamment le fait que les matrices $I, D, D^{\prime}$ sont linéairement indépendantes, on déduit que la composante neutre de l'isotropie du drapeau $\Omega$ est triviale.

Exemple 4. Soient $E, F$ deux $k$-espaces vectoriels de dimensions finies ; le groupe $\mathrm{Gl}(E) \times \mathrm{Gl}(F)$ agit sur $E \otimes F$. On a donc un morphisme $i: \mathrm{Gl}(E) \times \mathrm{Gl}(F) \rightarrow$ $\mathrm{Gl}(E \otimes F)$. On pose $\hat{G}=\mathrm{Gl}(E \otimes F)$ et $G=i(\mathrm{Gl}(E) \times \mathrm{Gl}(F))$. On a alors la

Proposition 6.5. - Dans la situation ci-dessus, toutes les faces sont pleines.

Démonstration. - La démonstration qui est analogue aux deux exemples précédents est ici omise.

\section{Résultats annexes}

7.1. - Soit $\mathbf{T}$ un tore, $\mathbf{D}$ un groupe semi-simple et $\mathbf{M}$ une $\mathbf{T} \times \mathbf{D}$-variété affine. Soit $\mathbf{P}$ un sous-groupe parabolique de $\mathbf{D}, \mathbf{H}$ son sous-groupe dérivé et $\mathbf{S}$ le centre connexe d'un sous-groupe de Levi de $\mathbf{P}$.

Notons $\rho: \mathbf{P} \times \mathbf{T} \longrightarrow \mathbf{S} \times \mathbf{T}$, l'application qui au couple $(p, t) \in \mathbf{P} \times \mathbf{T}$ associe le couple $(s, t) \in \mathbf{S} \times \mathbf{T}$ où $s$ vérifie $s^{-1} p \in \mathbf{H}$.

Proposition 7.1. - Avec les notations introduites ci-dessus, on suppose que $\mathbf{M}$ est factorielle.

Alors, il existe un ouvert non vide $\Omega$ de $\mathbf{M}$ tel que pour tout $x$ dans $\Omega$

$$
\operatorname{Ker}(\mathbf{S} \times \mathbf{T} \longrightarrow \operatorname{Aut}(\mathbf{M} / / \mathbf{H}))=\rho\left((\mathbf{P} \times \mathbf{T})_{x}\right) .
$$

Démonstration. - Notons $\pi: \mathbf{M} \longrightarrow \mathbf{M} / / \mathbf{H}$ l'application quotient. D'après les lemmes 3 et 2 , il existe un ouvert non vide $\Omega_{1}$ de $\mathbf{M} / / \mathbf{H}$ tel que pour tout $z$ dans $\Omega_{1}, \pi^{-1}(z)$ contient une unique orbite ouverte de $\mathbf{H}$. Quitte à remplacer $\Omega_{1}$ par un ouvert plus petit, on peut supposer de plus que pour tout $z$ dans $\Omega_{1},(\mathbf{S} \times \mathbf{T})_{z}$ est égal à $\operatorname{Ker}(\mathbf{S} \times \mathbf{T} \longrightarrow \operatorname{Aut}(\mathbf{M} / / \mathbf{H}))$ en vertu du lemme 5 .

L'ensemble $\Omega_{2}$ des points $x$ de $\mathbf{M}$ tels que la dimension de $\mathbf{H} . x$ soit maximale est un ouvert non vide de $\mathbf{M}$. Posons $\Omega=\Omega_{2} \cap \pi^{-1}\left(\Omega_{1}\right)$.

Soit $x \in \Omega$. Posons $z=\pi(x)$. Nous affirmons que :

$$
(\mathbf{S} \times \mathbf{T})_{z}=\{(s, t) \in \mathbf{S} \times \mathbf{T}:(s, t) . x \in \mathbf{H} . x\} .
$$

En effet, $(\mathbf{S} \times \mathbf{T})_{z}$ stabilise $\pi^{-1}(z)$ et permute les orbites de $\mathbf{H}$. Or, $\mathbf{H} . x$ est l'unique orbite ouverte de $\mathbf{H}$ dans $\pi^{-1}(z)$. Donc, $(\mathbf{S} \times \mathbf{T})_{z}$ stabilise $\mathbf{H}$. $x$.

Enfin, pour tout $(s, t) \in \mathbf{S} \times \mathbf{T}$, on a :

$$
\begin{aligned}
(s, t) . x \in \mathbf{H} . x & \Longleftrightarrow \exists h \in \mathbf{H} \quad \text { st } . x=h . x \\
& \Longleftrightarrow \exists h \in \mathbf{H} \quad(h s, t) . x=x \\
& \Longleftrightarrow(s, t) \in \rho\left((\mathbf{P} \times \mathbf{T})_{x}\right) .
\end{aligned}
$$


7.2. - On conserve les notations de la section 7.1. On suppose de plus que :

- D est un sous-groupe d'un groupe semi-simple $\hat{\mathbf{D}}$.

- T est le produit d'un tore $\mathbf{T}_{1}$ est d'un tore maximal $\hat{\mathbf{T}}$ de $\hat{\mathbf{D}}$.

- $\mathbf{M}$ est le produit d'une $\mathbf{T}_{1} \times \mathbf{D}$-variété $V$ et de $\hat{\mathbf{D}} / \hat{\mathbf{U}}$, où $\hat{\mathbf{U}}$ est un sousgroupe unipotent maximal de $\hat{\mathbf{D}}$.

- $\mathbf{T}_{1} \times \hat{\mathbf{T}} \times \mathbf{D}$ agit sur $\hat{\mathbf{D}} / \hat{\mathbf{U}}$ par $:(t, \hat{t}, d) \cdot \hat{d} \hat{\mathbf{U}}=d \hat{d}^{-1} \hat{\mathbf{U}}$.

Soit $\hat{\mathbf{B}}$ le sous-groupe de Borel de $\hat{\mathbf{D}}$ contenant $\hat{\mathbf{U}}$. Considérons l'application $\hat{\mathbf{D}}$-équivariante $q: \hat{\mathbf{D}} / \hat{\mathbf{U}} \longrightarrow \hat{\mathbf{D}} / \hat{\mathbf{B}}$.

Dans ce cas, la proposition 7.1 est complétée par la

Proposition 7.2. - Conservons les notations ci-dessus. Soit $x \in X$ et $y \in$ $\hat{\mathbf{D}} / \hat{\mathbf{U}}$. Alors, $\rho\left((\mathbf{P} \times \mathbf{T})_{(x, y)}\right)$ est isomorphe au quotient de $\left(\mathbf{P} \times \mathbf{T}_{1}\right)_{(x, q(y))}$ par son radical unipotent.

Démonstration. - Considérons la projection $p: \mathbf{P} \times \mathbf{T}_{1} \times \hat{\mathbf{T}} \longrightarrow \mathbf{P} \times \mathbf{T}_{1}$. Remarquons tout d'abord que comme chaque fibre de $q$ est isomorphe à $\hat{\mathbf{T}}, p$ induit un isomorphisme de $\left(\mathbf{P} \times \mathbf{T}_{1} \times \hat{\mathbf{T}}\right)_{y}$ sur $\left(\mathbf{P} \times \mathbf{T}_{1}\right)_{q(y)}$.

Montrons par ailleurs que $\rho$ induit un isomorphisme de $\left(\mathbf{P} \times \mathbf{T}_{1} \times \hat{\mathbf{T}}\right)_{y} /(\mathbf{P} \times$ $\left.\mathbf{T}_{1} \times \hat{\mathbf{T}}\right)_{y}^{u}$ sur $\rho\left(\left(\mathbf{P} \times \mathbf{T}_{1} \times \hat{\mathbf{T}}\right)_{y}\right)$.

Comme l'action de $\mathbf{P}$ sur $\hat{\mathbf{D}} / \hat{\mathbf{U}}_{\mathbf{D}}^{-}$est la restriction de celle de $\hat{\mathbf{D}}, \mathbf{P}_{y}$ est unipotent. Donc, $\mathbf{P}_{y}$ est inclus dans $\left(\mathbf{P} \times \mathbf{T}_{1} \times \hat{\mathbf{T}}\right)_{y}^{u}$. En particulier, le noyau de la restriction de $\rho$ à $\left(\mathbf{P} \times \mathbf{T}_{1} \times \hat{\mathbf{T}}\right)_{y}$ est inclus dans $\left(\mathbf{P} \times \mathbf{T}_{1} \times \hat{\mathbf{T}}\right)_{y}^{u}$. L'inclusion réciproque est vraie car $\rho\left(\left(\mathbf{P} \times \mathbf{T}_{1} \times \hat{\mathbf{T}}\right)_{y}^{u}\right)$ est un sous-groupe unipotent d'un tore : il est donc trivial.

Mais alors, sur le diagramme commutatif suivant :

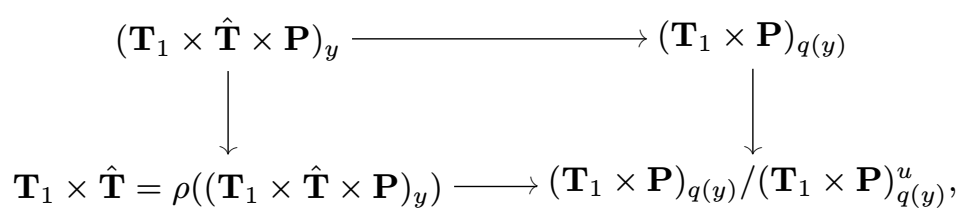

les flèches horizontales sont des isomorphismes. On obtient alors l'isomorphisme recherché par restriction à $\left(\mathbf{T}_{1} \times \hat{\mathbf{T}} \times \mathbf{P}\right)_{(x, y)}$ grâce à l'affirmation suivante : Soit $R$ un groupe résoluble connexe et $R^{\prime}$ un sous-groupe de $R$. Alors, $R^{\prime u}=R^{\prime} \cap R^{u}$. En effet, le sous-groupe $R^{\prime} \cap R^{u}$ est distingué dans $R^{\prime}$ et unipotent : il est donc inclus dans $R^{\prime u}$.

Par ailleurs, $R^{\prime} /\left(R^{\prime} \cap R^{u}\right)$ s'injecte dans $R / R^{u}$ qui est un tore. Donc, $R^{\prime} /\left(R^{\prime} \cap\right.$ $\left.R^{u}\right)$ est réductif et $\left(R^{\prime} \cap R^{u}\right) \subset R^{\prime u}$. 


\section{BIBLIOGRAPHIE}

[1] P. Belkale \& S. KumaR - «Eigenvalue problem and a new product in cohomology of flag varieties », Invent. Math. 166 (2006), p. 185-228.

[2] A. Berenstein \& R. SjamaAR - «Coadjoint orbits, moment polytopes, and the Hilbert-Mumford criterion », J. Amer. Math. Soc. 13 (2000), p. $433-466$.

[3] M. BRION - «Sur l'image de l'application moment », in Séminaire d'algèbre Paul Dubreil et Marie-Paule Malliavin (Paris, 1986), Lecture Notes in Math., vol. 1296, Springer, 1987, p. 177-192.

[4] _ «On the general faces of the moment polytope », Internat. Math. Res. Notices (1999), p. 185-201.

[5] A. G. ĖLAshvili - «Invariant algebras », in Lie groups, their discrete subgroups, and invariant theory, Adv. Soviet Math., vol. 8, Amer. Math. Soc., 1992, p. 57-64.

[6] W. Fulton - Introduction to toric varieties, Annals of Mathematics Studies, vol. 131, Princeton University Press, 1993, The William H. Roever Lectures in Geometry.

[7] G. Hochschild \& G. D. Mostow - « Unipotent groups in invariant theory », Proc. Nat. Acad. Sci. U.S.A. 70 (1973), p. 646-648.

[8] M. Kapovich \& J. J. Millson - «Structure of the tensor product semigroup », Asian J. Math. 10 (2006), p. 493-539.

[9] A. A. KLyachко - «Stable bundles, representation theory and Hermitian operators », Selecta Math. (N.S.) 4 (1998), p. 419-445.

[10] A. Knutson, T. TaO \& C. Woodward - « The honeycomb model of $\mathrm{GL}_{n}(\mathbb{C})$ tensor products. II. Puzzles determine facets of the LittlewoodRichardson cone », J. Amer. Math. Soc. 17 (2004), p. 19-48.

[11] D. LunA - «Sur les orbites fermées des groupes algébriques réductifs », Invent. Math. 16 (1972), p. 1-5.

[12] L. Manivel - «Applications de Gauss et pléthysme », Ann. Inst. Fourier (Grenoble) 47 (1997), p. 715-773.

[13] P.-L. Montagard - «Sur les faces du cône associé au pléthysme », Comm. Algebra 26 (1998), p. 2321-2336.

[14] L. NESS - «A stratification of the null cone via the moment map », Amer. J. Math. 106 (1984), p. 1281-1329, With an appendix by David Mumford.

[15] V. L. Popov \& E. B. VinBerG - «Algebraic Geometry IV », Encyclopedia of Mathematical Sciences, vol. 55, ch. Invariant Theory, p. 123-284, Springer-Verlag, 1991. 
[16] H. WeYL - « Das asymptotische Verteilungsgesetz der Eigenwerte linearer partieller Differentialgleichungen (mit einer Anwendung auf die Theorie der Hohlraumstrahlung) », Math. Ann. 71 (1912), p. 441-479.

[17] A. Zelevinsky - «Littlewood-Richardson semigroups », in New perspectives in algebraic combinatorics (Berkeley, CA, 1996-97), Math. Sci. Res. Inst. Publ., vol. 38, Cambridge Univ. Press, 1999, p. 337-345. 